\title{
The Super-Kamiokande Gadolinium Project
}

\author{
Hiroyuki Sekiya*i \\ Institute for Cosmic Ray Research, The University of Tokyo \\ E-mail: sekiyaeicrr.u-tokyo.ac.jp
}

The Super-Kamiokande Gadolinium (SK-Gd) Project is the upgrade of the SK detector via the addition of water-soluble gadolinium (Gd) salt. This modification will enable it to efficiently identify low energy anti-neutrinos. SK-Gd will pursue low energy physics currently inaccessible to SK due to backgrounds. The most important will be the world's first observation of the diffuse supernova neutrino background. On June 27 2015, the Super-Kamiokande Collaboration approved the SK-Gd project.

38th International Conference on High Energy Physics

3-10 August

2016

Chicago, USA

*Speaker.

${ }^{\dagger}$ Super-Kamiokande Collaboration 


\section{Supernova relic neutrinos}

Supernovae have been occurred since the beginning of the universe, and the neutrinos from all the supernovae have been accumulated as diffuse neutrinos in our universe, called supernova relic neutrinos(SRNs). SRNs would tell us the star formation history of the universe. Since reactor neutrinos and atmospheric neutrinos are dominant background below $10 \mathrm{MeV}$ and above $30 \mathrm{MeV}$ respectively, 10-30 MeV is an open window for the SRN measurement[1]. SK has been searching for SRN not only by only detecting prompt positron signals but also by coincidence detection of positrons and delayed $2.2 \mathrm{MeV}$ gammas from protons which captures neutrons, however it is still constrained by background because the efficiency of the $2.2 \mathrm{MeV}$ detection with Cherenkov lights is low. By adding $0.2 \%$ gadolinium sulfate into the $50 \mathrm{kton}$ water tank, this situation should be significantly improved [2] as shown in Figure 1. Gadolinium has a thermal neutron capture cross section of 49,000 barns (about 5 orders of magnitude larger than that of protons) and emits a gamma cascade of $8 \mathrm{MeV}$ that can be easily detected using Cherenkov light. In order to obtain a $90 \%$ efficiency for neutron capture, the Gd concentration should be $0.1 \%$, or $0.2 \%$ if $\mathrm{Gd}_{2}\left(\mathrm{SO}_{4}\right)_{3}$ is used, as illustrated in Figure 1. Figure 2 shows expected signal spectra for SRN models and
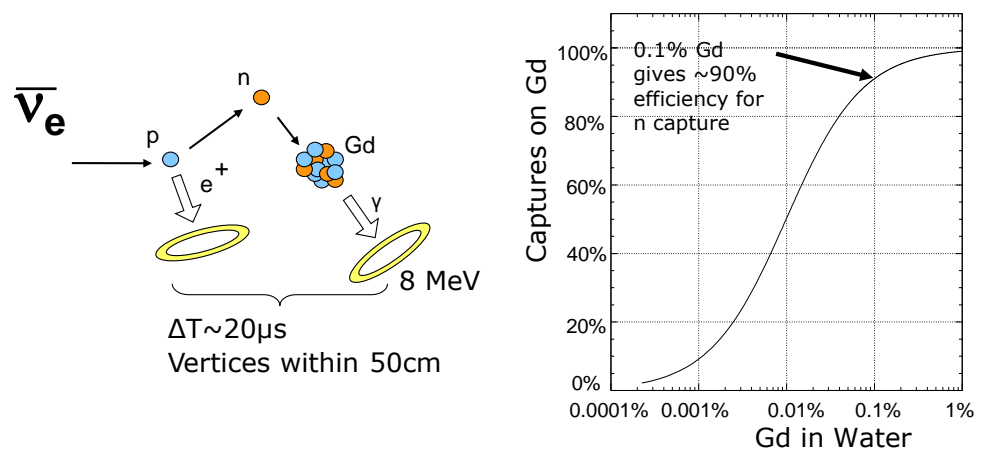

Figure 1: Neutron tagging with gadolinium (left), and capture efficiency as a function of Gd concentration (right).

conservative background estimation of atmospheric neutrinos with its component. Table 1 shows the expected number of events and background with 10 years observation. Here, we assume the $v_{\mu}$ CC background events are 1/4 of those of SK, $v_{e}$ CC background events are $2 / 3$, and NC background events are 1/3 with neutron tagging, respectively. Topological information of events should allow to reduce the background further, but it is not included in this estimation.

\section{EGADS}

In order to study the effect of dissolving Gd in the SK tank, an R\&D project called EGADS (Evaluating Gadolinium's Action on Detector Systems) has been running in the Kamioka mine. A new hall was excavated near SK and a $200 \mathrm{~m}^{3}$ stainless steel tank with ancillary equipment was constructed. The idea is to mimic the SK conditions inside the $200 \mathrm{~m}^{3}$ tank. The tank is equipped with a selective water filtration system that removes impurities while retaining the $\mathrm{Gd}$, a $15 \mathrm{~m}^{3} \mathrm{Gd}$ premixing and pretreatment plastic tank, and a device to measure the water attenuation length(called UDEAL). 

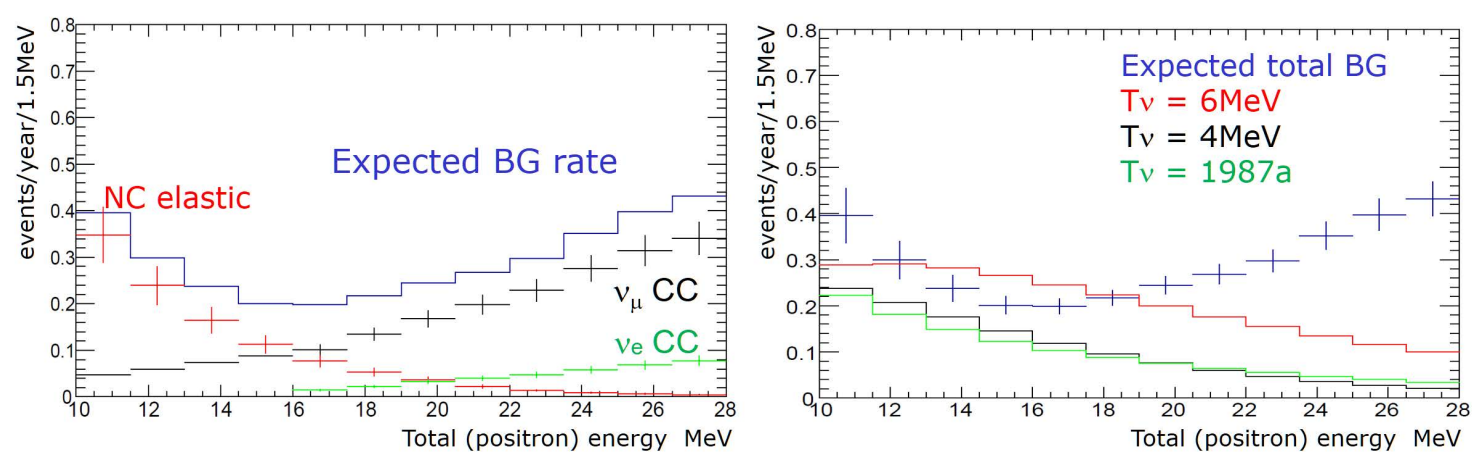

Figure 2: Expected background components of SK-Gd(left), and expected SRN spectra of some models(right).

\begin{tabular}{|c|c|c|c|c|}
\hline Model & $10-16 \mathrm{MeV}$ & $16-28 \mathrm{MeV}$ & Total & significance \\
\hline \hline$T_{\text {eff }}=8 \mathrm{MeV}$ & 11.3 & 19.9 & 31.2 & $5.3 \sigma$ \\
$T_{\text {eff }}=6 \mathrm{MeV}$ & 11.3 & 13.5 & 24.8 & $4.3 \sigma$ \\
$T_{\text {eff }}=4 \mathrm{MeV}$ & 7.7 & 4.8 & 12.5 & $2.5 \sigma$ \\
$T_{\text {eff }}=\mathrm{SN} 1987 \mathrm{a}$ & 5.1 & 6.8 & 11.9 & $2.1 \sigma$ \\
$\mathrm{BG}$ & 10 & 24 & 34 & - \\
\hline
\end{tabular}

Table 1: Expected numbers of SRN signals and backgrounds through SK-Gd 10 years observation

Figure 3 shows the history of the measured measured water transparency in $200 \mathrm{~m}^{3}$ tank since October, 2014. The four loadings are clearly identified. It demonstrates that transparencies can be comparable to those achieved at the pure-water SK's phases III and IV, and that in steady operation the measured transparencies are nicely stable at values very appropriate for physics analyses. The $\mathrm{Gd}_{2}\left(\mathrm{SO}_{4}\right)_{3}$ concentration in the $200 \mathrm{~m}^{3}$ tank is monitored by using an Atomic Absorption Spectrometer(AAS). Water samples are collected by a pump from three points in the detector, at three different height, using the same pipe as the UDEAL sampling. The measured concentration since November 2014 is also shown in Figure 3 The four loadings are clearly seen as steps in the concentration. The concentrations at all three sampling points are: 1) very close to each other, indicating that there is a homogeneous solution in the $200 \mathrm{~m}^{3}$ tank, and 2) stable between loadings or any other external intervention, demonstrating that there is no significant Gd loss during continuous water recirculation and purification.

\section{Implementation in Super-Kamiokande}

A $\mathrm{T} 2 \mathrm{~K}+\mathrm{SK}$ joint protocol is being established to take the decision about when to trigger the project and its implementation. It takes into account the needs of both experiments, the readiness of SK-Gd project, the T2K schedule, the J-PARC MR power upgrade and other miscellaneous elements. The current expected time of start is 2018. Respecting the SK detector probably the most critical item is its refurbishment of the tank. The scaling up of EGADS water system for SuperK-Gd is well under way. The current plan for the implementation of SK-Gd has three phases. 


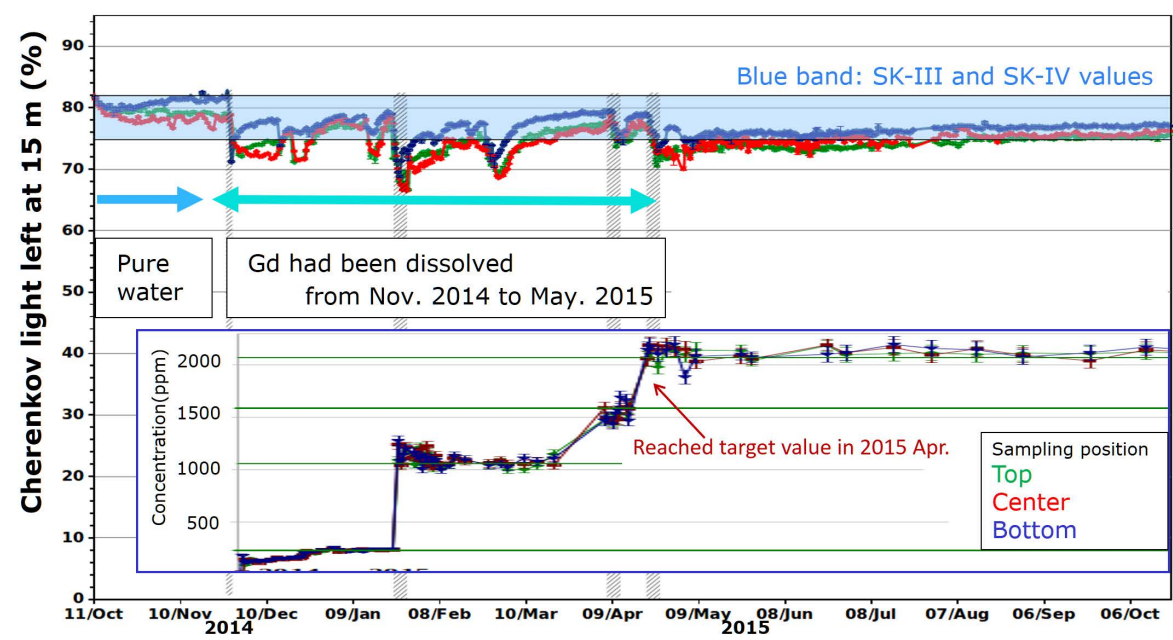

Figure 3: Percentage of Cherenkov light remaining for water inside the instrumented $200 \mathrm{~m}^{3}$ EGADS tank for increasing concentrations of gadolinium sulfate. The green, red, and blue lines correspond to data taken from the top, center, and bottom sample points of the $200 \mathrm{~m}^{3}$ tank, respectively, while the light blue band shows the range of ultra-pure water transparencies during SK-III and SK-IV. The inset is the gadolinium sulfate concentration history, as measured by the AAS, for the three sampling points in the top, center and bottom of the EGADS detector.

The first period is for the tank refurbishment, pure water fill, and recirculation of pure water in the detector until good water transparency is achieved. The second period is for loading $\mathrm{Gd}_{2}\left(\mathrm{SO}_{4}\right)_{3}$ up to $0.02 \%$, which corresponds to $\sim 50 \%$ neutron capture efficiency on Gd. We will run the detector for a while at this concentration in order to study backgrounds and neutron capture efficiency. In the third period we will add the remaining $\mathrm{Gd}_{2}\left(\mathrm{SO}_{4}\right)_{3}$ needed to achieve our full target loading, $0.2 \%$.

\section{References}

[1] S. Horiuchi et al., Phys. Rev. D79, 083013(2009).

[2] J. Beacom and M. Vagins, Phys. Rev. Lett. 93, 171101 (2004). 